\title{
Характеристики нанонаполнителя и межфазных областей в нанокомпозитах полимер/углеродные нанотрубки с эластомерной и стеклообразной матрицей
}

\author{
(2019 Л. Б. Атлуханова ${ }^{1}$, И. В. Долбин ${ }^{2}$, г. В. Козлов ${ }^{2 凶}$ \\ ${ }^{1}$ Дагестанский государственный медицинский университет \\ пл. Ленина, д. 1, 367000 Махачкала, Российская Федерация \\ ${ }^{2}$ Кабардино-Балкарский государственный университет им. Х. М. Бербекова \\ ул. Чернышевского, д. 173, 360004 Нальчик, Российская Федерация
}

\begin{abstract}
Аннотация. Целью настоящей работы является раздельное определение модуля упругости компонент нанокомпозитов полидициклопентандиен/многослойные углеродные нанотрубки, а именно, нанонаполнителя и межфазных областей. Для достижения этой цели использована микромеханическая модель.

Выполненные оценки продемонстрировали, что модуль упругости углеродных нанотрубок в полимерной матрице нанокомпозита, т. е., их агрегатов, приблизительно на два порядка меньше номинальной величины этого параметра для отдельной углеродной нанотрубки, тогда как модуль упругости межфазных областей примерно в два раза выше модуля упругости матричного полимера. Эти данные ясно демонстрируют некорректность применения номинальных характеристик нанонаполнителя, в частности, его модуля упругости, для определения соответствующих показателей нанокомпозита. Однако использование реальных величин модуля упругости агрегатов углеродных нанотрубок в рамках простого правила смесей позволяет достаточно точное описание этого параметра в случае нанокомпозитов. Важно отметить, что модуль упругости углеродных нанотрубок в эластомерной матрице существенно меньше этого параметра в стеклообразной матрице для одного и того же нанокомпозита. Это означает, что указанный параметр определяется не только размерами и структурой агрегатов нанонаполнителя, но также и другими факторами, в частности, жесткостью окружающей агрегат полимерной матрицы, эффективностью переноса приложенного к образцу механического напряжения от полимерной матрицы к нанонаполнителю и т. п.

Применение модифицированного правила смесей для описания модуля упругости нанокомпозитов показало, что включенный в него, так называемый, фактор эффективности длины в случае анизотропного нанонаполнителя существенно меньше (на несколько порядков) рассчитанного теоретически для углеродных нанотрубок, что особенно очевидно выражено в случае нанокомпозитов с эластомерной матрицей.

В качестве вывода укажем, что модуль упругости компонент нанокомпозита является сильной функцией их фазового состояния, а определение реальных характеристик этих компонент позволяет корректное применение простого правила смесей.
\end{abstract}

Ключевые слова: нанокомпозит, углеродные нанотрубки, полимерная матрица, модуль упругости, фазовое состояние, правило смесей.

\section{ВВЕДЕНИЕ}

В настоящее время углеродные нанотрубки считаются наиболее перспективным нанонаполнителем для полимерных нанокомпозитов в силу двух основных факторов: высокого продольного модуля упругости нанотрубок и

Козлов Георгий Владимирович, e-mail: i_dolbin@mail.ru их высокой степени анизотропии [1]. Однако на практике эти ожидания часто не оправдываются, поскольку в настоящее время предполагается [2], что для всех типов нанонаполнителя усиление нанокомпозитов реализуется не отдельными наночастицами, а их агрегатами. В случае нанокомпозитов полимер/углеродные нанотрубки нанонаполнитель формирует в полимерной мат-

Контент доступен под лицензией Creative Commons Attribution 4.0 License.

The content is available under Creative Commons Attribution 4.0 License. 
рице кольцеобразные структуры, внешне напоминающие макромолекулярные клубки [2], что резко снижает как модуль упругости [3], так и уровень реальной анизотропии нанотрубок по сравнению с номинальными [2]. Кроме того, в настоящее время теоретически [3] и экспериментально [4] показано, что модуль упругости межфазных областей нанокомпозитов существенно превышает соответствующий показатель для объемной полимерной матрицы и по абсолютной величине приближается к модулю упругости агрегатов нанонаполнителя. Это означает, что межфазные области являются таким же армирующим элементом структуры нанокомпозита, как и собственно нанонаполнитель [5]. Поэтому целью настоящей работы является оценка упругих характеристик агрегатов нанонаполнителя и межфазных областей нанокомпозитов полимер/углеродные нанотрубки с эластомерной и стеклообразной матрицей.

В настоящей работе экспериментальные результаты авторов [6] использованы для получения принципиально новой трактовки усиления нанокомпозитов полимер/углеродные нанотрубки с эластомерной и стеклообразной матрицей, которая отличается от предложенной в работе [6].

\section{ЭКСПЕРИМЕНТАЛЬНАЯ ЧАСТЬ}

В качестве нанонаполнителя использованы многослойные углеродные нанотрубки (МУНТ), имеющие внешний диаметр 15-20 nm, внутренний диаметр 5-10 nm и длину 0.5-20 $\mu \mathrm{m}$. Эти МУНТ были функционализированы нонборненом для повышения уровня межфазной адгезии полимерная матрица-нанонаполнитель. Содержание МУНТ составляло 0.05-0.40 mass \%. В качестве полимерной матрицы использован полидициклопентандиен (ПДЦПД) [6]. Для получения нанокомпозитов функционализированные МУНТ диспергировались в водном растворе ПДЦПД и подвергались обработке ультразвуком для улучшения диспергирования нанонаполнителя. Затем эта смесь перемешивалась с катализатором (дихлор-(3-метил-2-бутенилидин) бис-(три-циклофентил) фосфином рутения) до получения однородного раствора и сшивалась в течение 2 часов при 343 К и 1.5 часа при 443 K [6]. Механические испытания на одноосное растяжение выполнены на универсальной испытательной машине Instron 5569 согласно ASTM D638 (образцы типа V) при температуре 450 К и скорости ползуна $1 \mathrm{~mm} / \mathrm{min}$. Каждый результат был получен как усреднение данных четырех испытаний [6].
Динамический механический анализ (ДМА) выполнен с использованием прибора TA Instruments модели Q800 DMA. Образцы испытаны на растяжение с частотой $1 \mathrm{~Hz}$ в интервале температур 303-583 K при скорости нагрева $3 \mathrm{~K} / \mathrm{min}$.

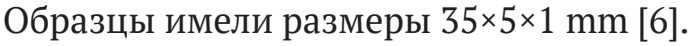

\section{РЕЗУЛЬТАТЫ И ИХ ОБСУЖДЕНИЕ}

Авторы [3] предложили следующее соотношение, позволяющее оценку модуля упругости агрегатов углеродных нанотрубок $E_{\mathrm{CNT}}$ и межфазных областей $E_{i f}$ с помощью графического построения:

$$
\frac{d E_{n}}{d \varphi_{n}}=\left(E_{i f}-E_{m}\right) \frac{d \varphi_{i f}}{d \varphi_{n}}+\left(\eta_{0} E_{\mathrm{CNT}}-E_{m}\right),
$$

где $E_{n}$ и $E_{m}-$ модуль упругости нанокомпозита и исходного матричного полимера соответственно, $\varphi_{n}$ и $\varphi_{i f}$ - объемное содержание нанонаполнителя и межфазных областей соответственно,, $\eta_{0}$ - параметр ориентации нанотрубок, принимаемый равным 0.38 [3]).

Величину $\varphi_{n}$ рассчитывали согласно формуле [6]:

$$
\varphi_{n}=\left[1+\left(\frac{\rho_{C N T}}{\rho_{m}}\right)\left(\frac{1-W_{n}}{W_{n}}\right)\right]^{-1},
$$

где $W_{n}$ - массовая доля нанонаполнителя, выраженная в долях единицы, $\rho_{\mathrm{CNT}}$ и $\rho_{m}$ - плотности углеродных нанотрубок и матричного полимеpa.

Величина $\rho_{\mathrm{CNT}}$ определена согласно уравнению [5]:

$$
\rho_{\mathrm{CNT}}=188\left(D_{\mathrm{CNT}}-d_{\mathrm{CNT}}\right)^{1 / 3}, \mathrm{~kg} / \mathrm{m}^{3},
$$

где $D_{\mathrm{CNT}}$ и $d_{\mathrm{CNT}}-$ внешний и внутренний диаметры углеродной нанотрубки, а величина $\rho_{m}$ принята равной $1200 \mathrm{~kg} / \mathrm{m}^{3}$ [6].

И, наконец, величина $\varphi_{i f}$ определяется с помощью следующего перколяционного соотношения [5]:

$$
\frac{E_{n}}{E_{m}}=1+11\left(\varphi_{n}+\varphi_{i f}\right)^{1,7},
$$

где отношение $E_{n} / E_{m}$ принято называть степенью усиления нанокомпозита.

На рис. 1 приведены зависимости $d E_{n} / d \varphi_{n}\left(d \varphi_{i f} / d \varphi_{n}\right)$, соответствующие уравнению (1), для нанокомпозитов ПДЦПД/МУНТ с эластомерной и стеклообразной матрицей. Как следует из данных рис. 1, эти зависимости достаточно хорошо аппроксимируются прямыми линиями, хотя и с сильно различающимся наклоном, что позволяет определить значения $E_{\mathrm{CNT}}$ и $E_{i f}$ для 
обеих серий рассматриваемых нанокомпозитов. Так, величина модуля упругости агрегатов (кольцеобразных формирований) МУНТ составляет $12.8 \mathrm{GPa}$ для нанокомпозитов ПДЦПД/МУНТ со стеклообразной матрицей и $2.15 \mathrm{GPa}$ - для этих же нанокомпозитов с эластомерной матрицей. Причина такого различия величин $E_{\mathrm{CNT}}$ очевидна из анализа уравнения (1) - более низкие значения модуля упругости матричного полимера $E_{m}$ в эластомерном состоянии и, следовательно, нанокомпозита $E_{n}$ (примерно, на два порядка) по сравнению со стеклообразной матрицей. Это означает, что ограничения на податливость агрегатов углеродных нанотрубок гораздо более жесткие для стеклообразной полимерной матрицы, чем для эластомерной. Таким образом, на величину $E_{\mathrm{CNT}}$ могут оказывать влияние не только характеристики собственно нанотрубок, но и свойства окружающей их полимерной матрицы. Отметим, что для нанокомпозитов поливиниловый спирт/углеродные нанотрубки авторы [3] получили $E_{\mathrm{CNT}}=71 \pm 55 \mathrm{GPa}$. Нетрудно видеть, что нижняя граница $E_{\mathrm{CNT}}$ для указанных нанокомпозитов близка к полученной в настоящей работе.

Расчет модуля упругости межфазных областей $E_{i f}$ согласно уравнению (1) дал следующие результаты: $E_{\text {iff }}=3.40 \mathrm{GPa}$ для нанокомпозитов ПДЦПД/МУНТ со стеклообразной матрицей и $E_{i f}=0.041 \mathrm{GPa}-$ с эластомерной. Как можно ви-

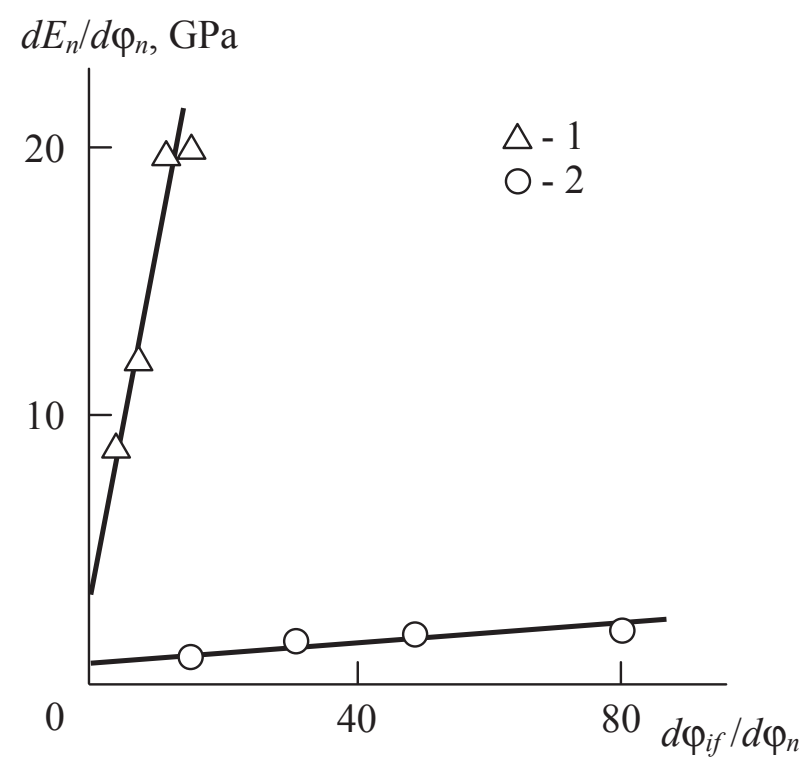

Рис. 1. Соотношение между производными $d E_{n} / d \varphi_{n}$ и $d \varphi_{i f} / d \varphi_{n}$ для нанокомпозитов ПДЦПД/МУНТ с эластомерной (1) и стеклообразной (2) матрицей [Fig. 1. The relationship between derivatives $d E_{n} / d \varphi_{n}$ and $d \varphi_{i f} / d \varphi_{n}$ for nanocomposites PDCPD/MCNT with elastomeric (1) and glassy (2) matrix] деть, в этом случае наблюдается различие величин $E_{\text {if }}$ практически на два порядка. Из уравнения (1) следуют две причины низкого значения $E_{\text {if }}$ для нанокомпозитов ПДЦПД/МУНТ с эластомерной матрицей: низкие величины $E_{m}$ и высокие значения $\varphi_{i f}$ Иначе говоря, предполагается, что межфазные области большой протяженности имеют рыхлоупакованную структуру и, как следствие, низкий модуль упругости. Отметим, что кристаллизующиеся межфазные области имеют величину $E_{i f}=46 \pm 5.5 \mathrm{GPa}$ [3], аморфные (для сшитого стеклообразного ПДЦПД) $E_{\text {if }}=3.40 \mathrm{GPa}$ и эластомерные - $0.041 \mathrm{GPa}$. Таким образом, в зависимости от фазового состояния межфазных областей их модуль упругости может различаться на три порядка. Это обстоятельство не означает снижения эффективности их армирующего действия. В настоящее время существует ряд моделей, например, модель Гута [7], уравнение Шеффера [2], перколяционная модель [5] и др., которые при расчете степени усиления нанокомпозитов не учитывают модули упругости нанонаполнителя и исходного матричного полимера, а оперируют другими параметрами (относительные доли нанонаполнителя и межфазных областей, реальная степень анизотропии нанонаполнителя и т. п.).

Для количественно проверки полученных указанным образом значений $E_{\mathrm{CNT}}$ может быть использовано хорошо известное правило смесей [8]:

$$
E_{n}=E_{\mathrm{CNT}} \varphi_{n}+E_{m}\left(1-\varphi_{n}\right) .
$$

На рис. 2 и 3 приведено сравнение рассчитанных согласно уравнению (5) и полученных экспериментально зависимостей модуля упругости $E_{n}$ от массового содержания нанонаполнителя $W_{n}$ для нанокомпозитов ПДЦПД/МУНТ со стеклообразной и эластомерной матрицей соответственно. Как можно видеть, получено достаточно хорошее соответствие результатов расчета согласно правилу смесей и экспериментальных данных (их среднее расхождение составляет $<1$ и $12.8 \%$ соответственно). Отметим, что использование в уравнении (1) номинальной величины $E_{\mathrm{CNT}}=E_{\mathrm{CNT}}^{0}$ $\approx 1000 \mathrm{GPa}$ [1] дает завышенные в несколько раз значения $E_{n}$ для рассматриваемых нанокомпозитов, особенно для нанокомпозитов ПДЦПД/ МУНТ с эластомерной матрицей. Кроме того, на рис. 2 и 3 наибольшее расхождение теории и эксперимента наблюдается при максимальной величине $W_{n}$. Следует указать, что уравнение (1) постулирует условие $E_{\mathrm{CNT}}=$ const. Однако изменение структуры агрегатов углеродных нанотрубок по мере вариации содержания нанонаполни- 


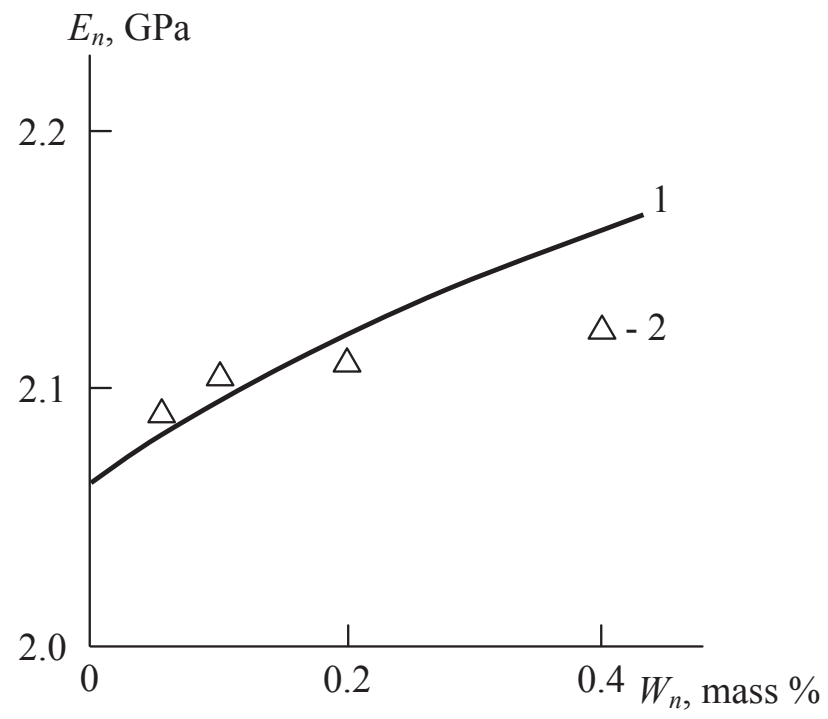

Рис. 2. Сравнение рассчитанной согласно правилу смесей (уравнение (5)) (1) и полученной экспериментально (2) зависимостей модуля упругости $E_{n}$ от массового содержания нанонаполнителя $W_{n}$ для нанокомпозитов ПДЦПД/МУНТ со стеклообразной матрицей

[Fig. 2. The comparison of calculated according to mixtures rule (the equation (5)) (1) and experimentally obtained (2) the dependences of modulus of elasticity $E_{n}$ on weigth contents of nanofiller $W_{n}$ for nanocomposites PDCPD/MCNT with glassy matrix]

теля предполагает изменение их эффективного модуля упругости. Так, авторы [9] показали, что эффективная величина $E_{\mathrm{CNT}}$ для нанокомпозитов полипропилен/углеродные нанотрубки снижается примерно в пять раз по мере роста $W_{n}$ в интервале 0.25-3.0 mass \%. Поэтому, если предположить снижение $E_{\mathrm{CNT}}$ в два раза для рассматриваемых нанокомпозитов в интервале $W_{n}=0.05-$ 0.40 mass \%, то это дает практически точное соответствие расчета согласно правилу смесей и экспериментальных данных.

Для описания повышения модуля упругости полимеров при введении анизотропных нанонаполнителей часто применяется модифицированное правило смесей [10]:

$$
E_{n}=\left(\eta_{L E} E_{\mathrm{CNT}}^{0}-E_{m}\right) \varphi_{n}+E_{m},
$$

где $\eta_{L E}$ - так называемый фактор эффективности длины, который увеличивается от 0 до 1 по мере роста аспектного отношения (отношения длина/диаметр) углеродных нанотрубок.

Для нанокомпозитов полимер/углеродные нанотрубки теоретический расчет дал $\eta_{L E}=3 / 8$ [3]. Однако оценки для рассматриваемых наноком-

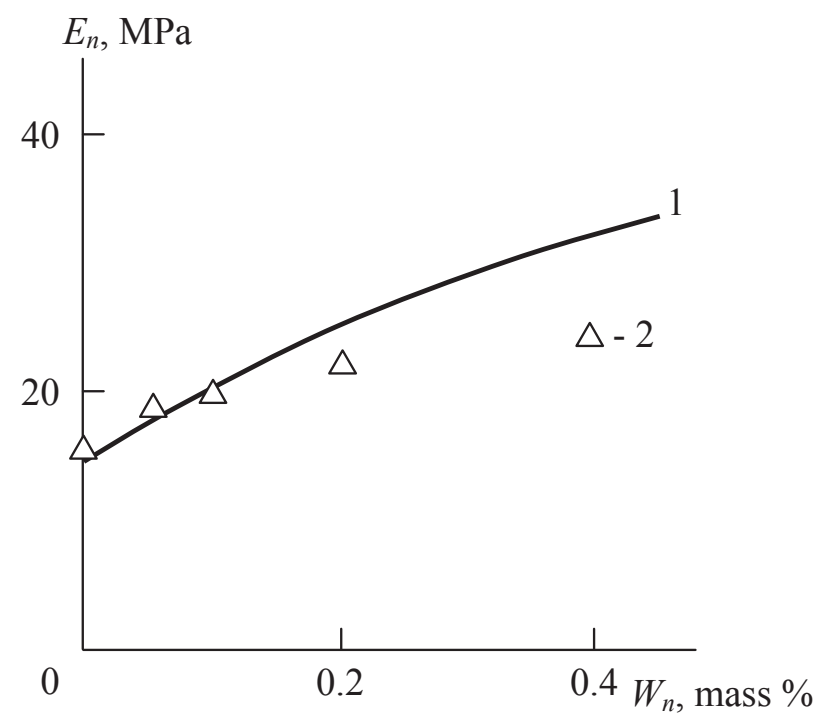

Рис. 3. Сравнение рассчитанной согласно правилу смесей (уравнение (5)) (1) и полученной экспериментально (2) зависимостей модуля упругости $E_{n}$ от массового содержания нанонаполнителя $W_{n}$ для нанокомпозитов ПДЦПД/МУНТ с эластомерной матрицей

[Fig. 3. The comparison of calculated according to mixtures rule (the equation (5)) (1) and experimentally obtained (2) the dependences of modulus of elasticity $E_{n}$ on weigth contents of nanofiller $W_{n}$ for nanocomposites PDCPD/MCNT with elastomeric matrix]

позитов ПДЦПД/МУНТ дают согласно уравнению (6) значения $\eta_{L E}=0.011-0.022$ в случае стеклообразной матрицы и $\eta_{L E}=0.00077-0.0024-$ в случае эластомерной, т. е. на несколько порядков ниже теоретического значения $\eta_{L E}$. В то же время из уравнений (5) и (6) следует $\eta_{L E}=E_{\mathrm{CNT}} / E_{\mathrm{CNT}}^{0}$, где средние значения $\varphi_{L E}$ равны 0.013 для случая стеклообразной матрицы и 0.00215 - для эластомерной. Это соответствие оценок фактора $\eta_{L E}$ в уравнении (6) и отношения $E_{\mathrm{CNT}} / E_{\mathrm{CNT}}^{0}$ предполагает совершенно другой физический смысл указанного фактора - он отражает снижение реального модуля упругости углеродных нанотрубок в полимерной матрице нанокомпозита вследствие их агрегации по сравнению с его номинальным значением.

\section{ЗАКЛЮЧЕНИЕ}

Таким образом, полученные в настоящей работе результаты предполагают, что нанокомпозиты полимер/углеродные нанотрубки усиливаются не собственно нанотрубками, а их агрегатами. Модуль упругости агрегатов углеродных нанотрубок зависит не только от их структуры, но 
и от податливости окружающей их полимерной матрицы нанокомпозита. Модуль упругости межфазных областей нанокомпозитов полимер/углеродные нанотрубки являются сильной функцией фазового состояния этих областей, и при переходе от эластомерных к кристаллическим областям величина указанного модуля может увеличиться на три порядка. Полученные в рамках модели [3] значения модуля упругости нанонаполнителя позволяют достаточно точный расчет соответствующего показателя для нанокомпозита в целом согласно простому правилу смесей.

\section{КОНФЛИКТ ИНТЕРЕСОВ}

Авторы декларируют отсутствие явных и потенциальных конфликтов интересов, связанных с публикацией настоящей статьи.

\section{СПИСОК ЛИТЕРАТУРЫ}

1. Moniruzzaman M., Winey K.I. Polymer nanocomposites containing carbon nanotubes // Macromolecules, 2006, v. 39(16), p. 5194. DOI: https://doi. org/10.1021/ma060733p

2. Schaefer D. W., Justice R. S. How nano are nanocomposites? // Macromolecules, 2007, v. 40(24), p. 8501. DOI: https://doi.org10.1021/ma070326w

3. Coleman J. N., Cadek M., Ryan K. P., Fonseca A., Nady J. B., Blau W. J., Ferreira M. S. Reinforcement of polymers with carbon nanotubes. The role of an ordered polymer intwrfacial region. Experimental and modeling // Polymer, 2006, v. 47(23), pp. 8556-8561. DOI: https://doi.org/10/1016/j.polymer.2006.10.014
4. Kozlov G. V., Yanovskii Yu. G., Zaikov G. E. Particulate-Filled Polymer Nanocomposites. Structure, Properties, Perspectives. New York, Nova Science Publishers, Inc., 2014. DOI: https://doi.org/10.1002/9783 527644346.ch3

5. Mikitaev A. K., Kozlov G. V., Zaikov G. E. Polymer Nanocomposites: Variety of Structural Forms and Applications. New York, Nova Science Publishers, Inc., 2008.

6. Jeong W., Kessler M.R. Toughness enhancement in ROMP functionalized carbon nanotube/polydicyclopentadiene composites. Chem. Mater., 2008. v. 20(22), p. 7060. DOI: https://doi.org/10.1021/cm8020947

7. Koerner H., Liu W., Alexander M., Mirau P., Dowty H., Vaia R. A. Deformation - morphology correlations in electrically conductive carbon nanotube - thermoplastic polyurethane nanocomposites // Polymer, 2005, v. 46(12), p. 4405. DOI: https://doi. org/10.1016/j.polymer.2005.02.025

8. Ahmed S., Jones F. R. A review of particulate reinforcement theories of polymer composites // J. Mater. Sci., 1990, v. 25(12), pp. 4933-4942. DOI: https://doi.org/10.1007/bf00580110

9. Aygubova A. Ch., Kozlov G. V., Magomedov G. M., Zaikov G. E. The elastic modulus of carbon nanotube aggregates in polymer nanocomposites. J. Characterization and Development of Novel Mater., 2016, v. 8(3), p. 227.

10. Khan U., May P., O’Neill A., Bell A.P., Boussac E., Martin A., Semple J., Coleman J. N. Polymer reinforcement usingliquid-exfoliated boron nitride nanosheets // Nanoscale, 2013, v. 5(3), pp. 581-587. DOI: https://doi. org/10.1039/c2nr33049k

\title{
The Physical Characteristics of Nanofiller and Interfacial Regions in Nanocomposites with Polymer/Carbon Nanotubes and Elastomeric Vitreous Matrix
}

\author{
(C) 2019 L. B. Atlukhanova ${ }^{1}$, I. V. Dolbin ${ }^{2}$, G.V. Kozlov ${ }^{2}$ \\ ${ }^{1}$ Dagestan State Medical University \\ 1, Lenina pl., 367000 Makhachkala, Russian Federation \\ ${ }^{2}$ Kh. M. Berbekov Kabardino-Balkarian State University \\ 173, Chernyshevski str., 360004 Nal'chik, Russian Federation
}

\begin{abstract}
Purpose. The purpose of this study is to separately define the elasticity modulus of nanocomposites polydicyclopentadiene/multilayer carbon nanotubes, specifically, of the nanofiller and interfacial regions. To achieve this we used a micromechanical model.
\end{abstract}

Georgii V. Kozlov, e-mail: i_dolbin@mail.ru 
Results. According to our estimates, the elasticity modulus of carbon nanotubes, or aggregates, in polymer matrix nanocomposite is approximately two orders of magnitude less than the nominal value of this parameter for separate carbon nanotube, while the elasticity modulus of interfacial regions is approximately two times than the elasticity modulus of the matrix polymer. Our data clearly demonstrates that it's incorrect to use the nanofiller's nominal characteristics, its elasticity modulus in particular, for determining the corresponding characteristics of the nanocomposite. Nevertheless, it's possible to use the real elasticity modulus values of carbon nanocomposite aggregates when using the simple rule of mixtures to determine this value for the nanocomposite with sufficient accuracy. It's important to note that the elasticity modulus of carbon nanotubes in an elastomeric matrix is much lower than the elasticity modulus in glassy matrix even if it's the same nanocomposite. This means that the given parameter is determined not only by the size and structure of the nanofiller aggregates, but also other factors, such as the stiffness of the polymer matrix around the aggregate, the effectiveness with which the polymer matrix transfers mechanical stress to the nanofiller, and so on.

When we used a modified rule of mixtures to determine the elasticity modulus of nanocomposites, we discovered that the so called length efficiency factor of carbon nanotubes, which is calculated using the modified rule of mixtures, is much (several orders of magnitude) lower than the one proposed theoretically, and that is especially evident in case of nanocomposites with an elastomeric matrix.

Conclusion. Thus, we conclude, that the elasticity modulus of nanocomposite components is a strong function of their phase state, and that it's possible to determine the real characteristics of these components by correctly using the rule of mixtures.

Keywords: nanocomposite, carbon nanotubes, polymer matrix, elasticity modulus, phase state, rule of mixtures.

\section{CONFLICT OF INTEREST}

The authors declare the absence of obvious and potential conflicts of interest related to the publication of this article.

\section{REFERENCES}

1. Moniruzzaman M., Winey K.I. Polymer nanocomposites containing carbon nanotubes. Macromolecules, 2006, v. 39(16), p. 5194. DOI: https://doi. org/10.1021/ma060733p

2. Schaefer D. W., Justice R. S. How nano are nanocomposites? Macromolecules, 2007, v. 40(24), p. 8501. DOI: https://doi.org10.1021/ma070326w

3. Coleman J. N., Cadek M., Ryan K. P., Fonseca A., Nady J. B., Blau W. J., Ferreira M. S. Reinforcement of polymers with carbon nanotubes. The role of an ordered polymer intwrfacial region. Experimental and modeling. Polymer, 2006, v. 47(23), pp. 8556-8561. DOI: https://doi.org/10/1016/j.polymer.2006.10.014

4. Kozlov G. V., Yanovskii Yu. G., Zaikov G. E. Particulate-Filled Polymer Nanocomposites. Structure, Properties, Perspectives. New York, Nova Science Publishers, Inc., 2014. DOI: https://doi.org/10.1002/9783 527644346.ch3

5. Mikitaev A. K., Kozlov G. V., Zaikov G. E. Polymer Nanocomposites: Variety of Structural Forms and Ap- plications. New York, Nova Science Publishers, Inc., 2008.

6. Jeong W., Kessler M.R. Toughness enhancement in ROMP functionalized carbon nanotube/polydicyclopentadiene composites. Chem. Mater., 2008. v. 20(22), p. 7060. DOI: https://doi.org/10.1021/ cm8020947

7. Koerner H., Liu W., Alexander M., Mirau P., Dowty H., Vaia R. A. Deformation - morphology correlations in electrically conductive carbon nanotube - thermoplastic polyurethane nanocomposites. Polymer, 2005, v. 46(12), p. 4405. DOI: https://doi. org/10.1016/j.polymer.2005.02.025

8. Ahmed S., Jones F. R. A review of particulate reinforcement theories of polymer composites. J. Mater. Sci., 1990, v. 25(12), pp. 4933-4942. DOI: https:// doi.org/10.1007/bf00580110

9. Aygubova A. Ch., Kozlov G. V., Magomedov G. M., Zaikov G. E. The elastic modulus of carbon nanotube aggregates in polymer nanocomposites. J. Characterization and Development of Novel Mater., 2016, v. 8(3), p. 227.

10. Khan U., May P., O’Neill A., Bell A.P., Boussac E., Martin A., Semple J., Coleman J. N. Polymer reinforcement using liquid-exfoliated boron nitride nanosheets. Nanoscale, 2013, v. 5(3), pp. 581-587. DOI: https://doi. org/10.1039/c2nr33049k 
Атлуханова Луиза Бремовна - к. п. н., доцент кафедры биофизики, информатики и медаппаратуры, Дагестанский государственный медицинский университет, Махачкала, Российская Федерация; e-mail: bremovna77@mail.ru. ORCID iD: 0000-0002-5341-3349.

Долбин Игорь Викторович - к. х. н., доцент кафедры органической химии и высокомолекулярных соединений, Кабардино-Балкарский государственный университет им. Х.М. Бербекова, Нальчик, Российская Федерация; e-mail: i_dolbin@mail.ru. ORCID iD: 0000-0001-9148-2831.

Козлов Георгий Владимирович - с. н. с., Кабардино-Балкарский государственный университет им. Х.М. Бербекова, Нальчик, Российская Федерация; e-mail: i_dolbin@mail.ru.ORCID iD: 00000002-9503-9113.
Luiza B. Atlukhanova - Cand. Sci. (Ped.), Associate Professor of the Department of Biophysics, Informatics and Medical Devices, Dagestan State Medical University, Makhachkala, Russian Federation; e-mail: bremovna77@mail.ru. ORCID iD: 0000-0002-5341-3349.

Igor V. Dolbin - Cand. Sci. (Chem.), Associate Professor of the Department of Organic Chemistry and High-Molecular Compounds, Kh. M. Berbekov Kabardino-Balkarian State University, Nal'chik, Russian Federation; e-mail:i_dolbin@mail.ru.ORCID iD: 0000-0001-9148-2831.

Georgii V. Kozlov - Management of Research and Innovation Activities, Kh. M. Berbekov KabardinoBalkarian State University, Nal'chik, Russian Federation; e-mail: i_dolbin@mail.ru. ORCID iD: 00000002-9503-911 $\overline{3}$. 